\title{
The Graphic Communication Actor: Generating Visual Rhetoric for Development Initiatives
}

\section{Ebigbagha Zifegha Sylvester}

Department of Fine and Applied Arts Niger Delta University, Wilberforce Island, Bayelsa State, Nigeria Email: ebigbaghazsylvester@yahoo.com

\author{
Article History \\ Received: December 14, 2019 \\ Revised: January 7, 2020 \\ Accepted: January 14, 2020 \\ Published: January 16, 2020 \\ Copyright (C) 2020 ARPG \& \\ Author \\ This work is licensed under the \\ Creative Commons Attribution \\ International \\ (c) (1) CC BY: Creative \\ Commons Attribution License \\ 4.0
}

\begin{abstract}
The art of using language for public expression in order to persuade target audience to support development initiatives is a key reason for graphic communication. This requires communication actors particularly, the graphic encoder to know salient input and output variables of communication for effective mediation. However, the prevalence ignorance of these variables, often results in ineffective media production that is counter-productive to development. Therefore, this paper focused on production of practical rhetoric in graphic language for development programmes. The paper employed the critical-historical-analytic examination and content analysis methods. It introduced the reader to the need for practical rhetoric in visual communication. Furthermore, it highlighted the salient input and output variables that the graphic communication actor need be conversant with in order to produce visual rhetoric, using the McGuire's Communication/persuasion Matrix. And it exemplified graphic media that result from application or neglect of the knowledge of the variables. The paper found that consideration of the variables afforded production of effective rhetoric in graphic language. The paper ended with the need for graphic encoders to internalize knowledge of the input and output variables and utilize it during the process of media production to generate visual rhetoric with desired effect.
\end{abstract}

Keywords: Rhetoric; Communication actor; Development initiative; Graphic communication; McGuire's communication/persuasion matrix.

\section{Introduction}

Globally, graphic language is often employed to share prime messages and supporting information of development programmes. This is hinged on the necessity to inform, mobilize, and persuade target audience to actively participate in development initiatives, which could readily be seen in campaigns on agriculture, education, and health, to mention a few. So, rhetoric in graphic communication is crucial for the success of development efforts.

Nevertheless, the art of generating and utilizing graphic language for public persuasion is hinged on collaboration among communication actors, which include members of the media team (a group of subject specialists, assigned with the responsibility to effectively communicate development messages with target audience) during the media production process in a given campaign. Within this collaborative efforts, the graphic encoder (person assigned to perform the task of transforming development ideas into graphic language), is required to have a comprehensive knowledge of input and output variables of communication in order to function effectively (Ejembi, 1989; citing Wright (1981).

However, there is prevalence ignorance of these independent and dependent variables by most communication actors including the graphic encoder. The result has often been neglect or obtrusive intrusion upon roles, activities and functions of different communication actors, particularly, the mediation role of the graphic encoder, and other members of the media team during media production process in communication campaign. This is a common situation in Nigeria, whereby anybody could be assigned to perform graphic encoding task that usually results in the production of ineffective graphic media with grave consequences for development (Ebigbagha, 2016). So, it is needful for the graphic encoder to know the input/ independent and output/mediating variables of communication and persuasion in order to mediate effectively during media production process and generate informative and persuasive graphic language.

Proper mediation of the graphic encoder during media development, usually culminates in generation of rhetorical information, education and communication (IEC) materials. However, the level of mediation/intervention of the graphic encoder is immensely determined by the communication practices and development paradigms adopted by the media requesting agency/ Source. The adoption of Modernization/Dependency concept of development that employs the hierarchical or Top-down/One-way communication approaches, often inhibits proper mediation role of the graphic encoder because the Source controls the entire communication development process. 
Conversely, the Source's adoption of Another Development/Multiplicity concept of development that uses the Participatory/Two-way communication practices, usually occasions effective graphic mediation because the Source engages the participation of all other communication actors, including the graphic encoder and target audience.

Therefore, effective graphic encoder's mediation, hinged on in-depth knowledge of input or independent communication variables, and output, dependent or mediating persuasion variables are prospective of practical rhetoric in graphic communication. Towards this end, we are going to critically examine variables for practical rhetoric in graphic communication, and present examples of practical rhetoric in visual communication hereunder.

\section{Variables for Practical Rhetoric in Graphic Communication}

Many input and output variables are necessary to create graphic language that communicate messages that effectively persuade target audience. This requires knowledge of how target audience process information and response to graphic treatment of development messages. In order to achieve this, it would be necessary to consider a general framework that is coalesce of communication and persuasion variables. An important model to guide our discussion in this respect, is the McGuire communication/persuasion matrix.

\subsection{The McGuire's Communication/Persuasion Matrix}

McGuire (1989), McGuire (1999) describes/prescribes the way target audience processes information and response to persuasive communication. He presented a communication and persuasion matrix of input/independent, and output/dependent or mediating variables (Figure 1).

The model identifies five communication components - Source, Message, Medium, Receiver, and Effect - with many input or independent variables, from which persuasive communication can be constructed. In addition, thirteen output steps - mediating and dependent variables - are stated, the evocation of which enhances persuasive impact.

\subsubsection{Source as Independent Variable for Rhetoric in Graphic Communication}

The communication process usually begins with the input/independent variable - the Source, which could be an individual, non-governmental, governmental, international or multinational organisation, or a combination of any of these organisations. McGuire (1999), pin pointed eleven factors of the Source that need be considered when constructing persuasive communication. These are credibility, expertise, trust, attractiveness, similarity, familiarity, neoteny, power, control, scurrility, and concern. When these are interrogated with the thirteen persuasion output variables: exposure, attention, liking, comprehension, cognitive elaboration, skill acquisition, agreement,

Figure-1. The communication/persuasion (input/output) matrix as an aid in constructing persuasive communication McGuire (1999) from Machado (2002)

\begin{tabular}{|c|c|c|c|c|c|c|c|}
\hline \multicolumn{8}{|c|}{ Communication Components Inputs: Independent Variables } \\
\hline \multirow{5}{*}{$\begin{array}{l}\text { Persuasion } \\
\text { Output } \\
\text { Mediating and } \\
\text { Dependent Variables }\end{array}$} & \multicolumn{3}{|c|}{ SOURCE } & \multirow{2}{*}{$\begin{array}{l}\text { MIESSAGE } \\
\text { Arguments }\end{array}$} & \multirow{2}{*}{$\begin{array}{l}\text { CHANNEL } \\
\text { (Medium) } \\
\text { Modality }\end{array}$} & \multirow{2}{*}{$\begin{array}{c}\begin{array}{c}\text { RECEIVER } \\
\text { (Audience) }\end{array} \\
\text { Demographics }\end{array}$} & \multirow{2}{*}{$\begin{array}{c}\text { RESPONSE } \\
\text { TARGET } \\
\text { Purchasing }\end{array}$} \\
\hline & Credibility & Attractiveness & Power & & & & \\
\hline & & Similarity & Control & Inclusions & Context & Personality & Voting \\
\hline & Expertise & Familiarity & Scurrility & Orderings & Nonverbal & Ability & Violence \\
\hline & Trust & Neoteny & Concern & Discrepancy & & & \\
\hline & & & & Style & & & \\
\hline $\begin{array}{ll}\text { 1. } & \text { Exposure } \\
\text { 2. } & \text { Attention } \\
\text { 3. } & \text { Liking } \\
\text { 4. } & \text { Comprehension } \\
\text { 5. } & \text { Cognitive } \\
\text { 6. } & \text { Elaboration } \\
\text { 7. All Acquisition } \\
\text { 8. Agreement } \\
\text { 9. } \text { Retriery Storage } \\
\text { 10. Decision Making } \\
\text { 11. Acting on } \\
\text { 12. } \text { Decision } \\
\text { 13. Pognitive } \\
\text { 13. Proselytizing }\end{array}$ & & & & & & & \\
\hline
\end{tabular}

- The column heading are the categories and subcategories of inputs (independent variables) out of which the persuasive communication can be constructed.

- The row headings are the output steps (mediating and dependent variables) the evocation of which enhances persuasive impact.

Memory storage, retrieval, decision making, acting on decision, cognitive consolidation, and proselytizing; a clear orientation for visual rhetoric is afforded.

Credibility, expertise and trust of the Source are salient points to be thoroughly considered in constructing persuasive communication. The target audience need believe that the source and its ideas as well as products are true, dependable and reliable. A credible source with immense expertise and trustworthy ideas and products would potentially attract target audience and constitute a virile structure for persuasive form generation. The genuineness, 
proficiency and technical dexterity as well as the believability of the Source in knowledge production and product development are formidable ingredients that are critical to creating persuasive messages. Contrarily, a Source bereft of credibility, expertise and trustworthiness would often be taken for granted, doubted and likely ignored. It is a common experience that products and ideas of mediocrity and amateurism are usually greeted with much scepticism, uncertainty, unwillingness or delayed response when compared with products of great expertise, credibility and trust. So, in configuring graphic messages, it is crucial to interrogate each input variable with the output variables. For example, what areas of credibility, expertise and trust of the Source could the target audience be exposed to in order to attract their attention and stimulate interest as well as generating liking and enhancing comprehension? The portrayal of a high degree of credibility, expertise, and trust of Source, products and ideas are indispensable to formulation of practical rhetoric in visual language for commercial, and institutional advertising purposes.

The establishment of a common ground is paramount to creating persuasive messages. McGuire (1989), McGuire (1999), described/prescribed attractiveness, similarity, familiarity and neoteny as input variables of the Source, which are pivotal for successful generation of practical rhetoric in visual communication. Attractiveness is a key purpose of communication in visual language, whether for predominantly aesthetic or utility products as in fine, and applied arts respectively. This could readily be seen in packaging of industrial, and manually generated products, whereby pleasing appearance is diligently crafted to arrest attention and stimulate interest of target audience for prompt response. This is in consonance with the universal principle of Attractive Bias, which states that attractive products are generally much more accepted and seemingly easier to utilize than less attractive ones Lidwell et al. (2013). Also, similarity, and familiarity between the Source and the target audience affords acceptance because it presents a known and shared reality; particularly when the reality associated with the Source, produces pleasurable experience in the life of target audience. This is hinged on the premise that learning or behaviour is conditioned, reinforced, and generalized when stimulus offers satisfactory experience (David, 2015). In addition, the Source should be presented in timeless freshness and potentials that sustain target audience support. Messages and products should be infused with neoteny, whereby, effervesces of juvenility is exuded even though the message/product is not new. Therefore, it is salient not to organize visual expressions in a grotesque, different, strange, and ephemeral manner that presents content and context with meanings that are parallel to Source's desired communication aim and target audience information needs.

Moreover, the power, control, scurrility, and concern of the Source are critical input variables to consider in order to create practical visual rhetoric. The ability of the Source to occasion desired outcomes with high credibility, responsibly taking charge of situation/activities in order to produce an overall salutary effect, utilizing language that is void of vulgarism, and express concern which support user needs are critical features of the Source that need be emphatically expressed. These are attractive and reliable characteristics that are salient to persuade target audience for support of development ideas or commercial products.

Fundamentally, the age, gender, socio-economic status, ethnicity, expertise, similarity, familiarity, concern, etc. of the communicator affect the extent to which the target audience are persuaded. This is because these aspects of the source give credibility, attractiveness and power to the receivers.

\subsubsection{Message as Independent Variable for Rhetoric in Graphic Communication}

A critical independent variable for practical rhetoric in visual communication is the message - content. McGuire (1999), prescribed that arguments, inclusions, orderings, discrepancy, and style of the message need be examined in order to create visual rhetoric. The perspectives presented in a given message should be understood by the target audience to be of superior value and of immense benefit that would translate to a better and higher standard of living. Also, it is needful to consider what the message is consists of - what would be omitted or included, how could it be depicted, are there variations, and in what manner could it be expressed?

The manner of expression could introduce technical, syntactic, semantic, pragmatic, and aesthetic/arousal uncertainties during the encoding process of ideas/message into graphic language, which often occasion a misleading misrepresentation, misunderstanding, and misinterpretation. For example, not applying the principle of Chunking: grouping related pieces of information to depict prime messages and supporting information would likely pose syntactic and semantic challenges to target audience. The style a message is expressed is essential because content attributes are capable of determining behaviour of receivers - negative or positive Ebigbagha (2019) citing Watt (1979). So, messages need to represent target audience based on objective measures through action research on the topic, pre-test, and evaluation during the media production process Ebigbagha (2016); citing Ejembi (1989); Bowers (1973).

Nevertheless, when the input variables: arguments, inclusions, orderings, discrepancy, and style of the message are questioned in juxtaposition with the output variables, a pathway for visual rhetoric is created. For examples, how could the message - its content, be configured to get maximally exposed, attract attention, liked, understood, memorized, and cognitively elaborated by receivers? How could the content of the message be transformed for target audience to acquire desired skill, support agreement, store and retrieve it from memory, make and act on decision, consolidate it cognitively, and proselytize as desired? These interrogations on the message during media development process often engenders production of visual rhetoric.

\subsubsection{Channel as Independent Variable for Rhetoric in Graphic Communication}

A paramount variable in the communication process, is channel. It carries the message in the communication process. McGuire (1999), states that particular attention should be devoted to modality, context, and nonverbal activities, functions and processes when producing/utilizing the input variable - channel. When these independent 
variables of communication are engaged with the dependent variables of persuasion: exposure, attention, liking, comprehension, cognitive elaboration, skill acquisition, agreement, memory storage, retrieval, decision making, acting on decision, cognitive consolidation, and proselytizing a potently persuasive channel emerges. For instance, what channel could best convey the message with utmost exposure, greatest attention, and liking of target audience?

From the above, media analysis is salient to launching visual rhetoric. It requires examination of the features and capabilities of the medium, its appropriateness and appeal to target audience as well as its potentials to suitably present the message as planned. Messages are well communicated by both verbal and non-verbal channels; however, media, which include variables that are audio/visual, written/spoken, verbal/nonverbal, vocalic/visual non-verbal messages have different effect in various context.

According to Anonymous (2007), media analysis is done by first deciding on the target audience, using, say the 'TGI', 'NRS' and other survey instruments to decide the most appropriate media to reach target audience. It is this consideration of appropriateness of medium in order to reach the targeted audience that resulted in the distribution of billboard posters to most Nigeria Universities on the Delayed Sexual Debut Campaign; which is adjudged a success by the NARHS evaluation (Society for Family Health, 2006).

To neglect a thorough consideration of the appropriateness of a medium to a given target audience, is an action oriented towards communication failure. This is because the medium is liable to noise that is capable of destroying the message and invariably, the entire communication process; and a wrong choice of medium could impede desired communication outcome. For this reason, advertising agencies employ media analysts, who decide what is the most suitable medium or combination of media for a given message they would want to communicate.

\subsubsection{Target Audience as Independent Variable for Rhetoric in Graphic Communication}

Target audience conditions are critical to generating practical rhetoric in visual communication. McGuire (1999), prescribed demographics, personality, lifestyle and ability as a set of independent variables of target audience that need be considered in order to communicate with target audience effectively and persuasively too. This requires audience research to find out the characteristics, behaviour, attitudes and values as well as likes and dislikes of the targeted population, which affect the way messages are received and perceived (Ebigbagha, 2016b). Target audience research is done using survey instruments such as the NAHRS, 'TGI' ratings figures and other data from 'BARB', and from 'Gallup' to mention a few.

The aforementioned independent variables of the target audience, when examined in the light of the thirteen persuasion output variables: exposure, attention, liking, comprehension, cognitive elaboration, skill acquisition, agreement, memory storage, retrieval, decision making, acting on decision, cognitive consolidation, and proselytizing; messages constructed thereof, are prospective of generating persuasive impellents. This kind of messages, would certainly be consistent with the knowledge, aptitude and practices of the target audience that is liked, understood and usually responded to as desired for a long time. This affords a change in target audience behaviour because messages are planned and communicated for long term effects rather than immediate effects, which are lesser in effectiveness in relation with remembering and persuasion

The need to know the target audience is important in all forms of communication whether at the level of interpersonal communication between, for example, teachers and learners, graphic designers and clients, doctors and patients or mass communication in support of development programmes as in the campaign on HIV/AIDS between the source and the target audience.

\subsubsection{Response Target as Independent Variable for Rhetoric in Graphic Communication}

Every communication is embarked upon in order to generate a response, result or achieve something. This is the Response Target - destination or effect, which is a communication variable that has to do with the type of target behaviour at which the communication is directed (McGuire, 1999). This includes immediate or long term change, or change on a specific issue for which the communication was occasioned. Furthermore, he described/prescribed purchasing, violence and voting as independent variables of communication that could be considered.

Whatever the targeted response is - purchasing, violence, voting, or change of behavioral pattern, messages that would persuade receivers to elicit desired feedback, need be configured to accommodate the thirteen mediation variables of persuasion already mentioned.

It is important to get feedback on target response for subsequent communication efforts, so that mistakes are corrected or avoided, and weak points could be strengthened. This is the reason broadcasters use the services of 'BARB' to gain feedback in form of ratings when they transmit a programme. Also, advertising agencies use a variety of services such as 'Gallup' to find out the effectiveness of the campaign on products, and the 'NARHS' was used to find out the effectiveness of the campaigns on HIV/AIDS in the Delayed Sexual Debut Campaign. Conversely, it was not done in the Use Condom Campaign and the result was ineffective communication outcomes (Ebigbagha, 2016).

Having discussed the input/independent variables of communication, been interfaced with the thirteen persuasive variables superficially, we would now consider in more detail, the thirteen persuasion outputs/mediating or dependent variables of the communication/persuasion matrix. These are:

i. Exposure, this is the first crucial step necessary for attitude change/information processing to occur. Before the target audience is involved in decision-making on a given message, he/she must be exposed to the message, which is then processed before he/she ascent through the stages of attitude change (Machado, 2002). 
ii. Attention, even, with exposure to the message, attention is not guaranteed. The human mind accepts only a small portion of the large number of information it receives. Therefore, messages should be organised in a way that attracts attention, which is necessary for attitude change to occur.

iii. Liking, is to become interested in the communication. For target audience to be persuaded, they need to develop interest on the message. So it is necessary to make messages interesting or likeable to target audience.

iv. Comprehension, this requires the target audience to grasp the meaning and implications of the message communicated. Apart from been understood, it must be able to generate deep thought or contemplated about. McGuire discussed abstraction, encoding and meaning systems as construct that affect comprehension. Therefore, graphic messages should be configured in a way that facilitates understanding and thinking about what is presented because it supports persuasion.

v. Cognitive elaboration, is critical thinking about what is communicated; it is closely related with comprehension. It holds that communication should be carried out in a way that stimulates critical and imaginative thoughts. This helps to persuade target audience, so graphic treatment of messages should provoke thinking.

vi. Skill acquisition is the skills target audience acquire by learning how to do what is communicated; as this is done, target audience are persuaded.

vii. Agreement is how believable or valid a message is. After a full grasp of what is communicated, an opinion must arise about the persuasive message whether it is believable, valid or not. Agreement with the message can be influenced by a number of factors such as previously held beliefs (internal) and the perception of the source as being credible or the type of appeal used (external). This step of agreement is also referred to as yielding/attitude change (McGuire, 1989).

viii. Memory storage, is to commit accepted message into the preconscious level of the mind, which can be recalled at will. This is necessary because sometime may elapsed between when a message is disseminated and when it is adopted/utilized. Therefore, development messages should be encoded in a way that would facilitate retention. However, messages committed to memory can still be liable to decay because delayed processing affects the storage of content as well as reprocessing of message.

ix. Retrieval is the act of information search and fetch or recall. It is closely related to memory storage but refers to the search strategies, which gather information from the cognitive storage systems. This could be organised in different ways, depending on whether the cognitive domain surrounding the development message is organised into a tree diagram, a matrix, pushdown lists, etc. There would be differences in the type of search strategies the target audience would and should use to bring this informational domain to bear on choices made on a given development idea (Machado, 2002). So, the search strategies, which would aid recall and rapid adoption of development messages by target audience should be taken into account in the graphic encoding process.

x. Decision-making is arrived at on the basis of the retrieval, after which the receiver makes a decision/choice from the available options. This decision can be made using different processes or 'choice strategies' including 'elimination-by-aspects' strategy, heuristics and the 'one-dimension-at-a-time' model (Machado, 2002).

xi. Acting on decision, is behaving in accord with prior firmness of conviction. It refers to actions and appropriate response taken on development messages. However, Blair et al. (1975) and Machado (2002) notes that action do not necessarily follow attitude as research has shown, despite the fact that an individual can experience the entire process as he/she passes through it. People always say one thing and do another because acting on decisions are influenced by imitation of others, peer groups, role models, family ties and society, among others. However, interest, which is a positive attitude that causes a person to seek more activities in a given area, when sufficiently stimulated would facilitate acting on decision, which is important in commercial and development communication.

xii. Cognitive Consolidation is the sustainability and reinforcement of behaviour from pleasant experiences, which result from knowing, understanding or learning a given development idea and acting on decision. The satisfaction derived from previous acting on decision based on stimulated interest would often lead to a repeat of such decisions whenever the need arises; and

xiii. Proselytizing is the expression of a previous pleasant experience in order to persuade someone to join in trying or acting on decision in the same way as previously done. This is based on previous knowledge, belief and confidence in a product, that the result would be as satisfying as expected. Therefore, it is salient to make graphic language for development communication persuasive, so that, it would result in this kind of desirable behaviour from target audience.

From the foregoing, McGuire shows the input and variables of communication and persuasion respectively that are important in planning practical rhetoric in graphic communication. Although it was originally intended to be employed in the field of commercial advertisement, it is equally useful in the field of development communication. These require communication strategies that persuade target audience to take deliberate action: to buy a given product, as in commercial advertising or to change a particular behaviour/adopt development ideas, as in development communication/ institutional advertising.

Also, the model is useful to measure the effectiveness of communication campaign by using input and output variables. It brings together multiple elements to be considered in the construction and evaluation of visual rhetoric, which can help to reinforce the communication strategy. Machado (2002) states that the model is a complete how-to- 
guide for the creation of persuasive message aimed at inducing an attitude change and/or a purchase action. So, to plan persuasive communication that requires collaborative efforts, these stages in the cognitive process could be very useful.

Nevertheless, the model has been criticized for presenting the human mind as if it were a programmed machine that follows a given order whenever it is confronted with making a choice. Not all change of attitude or decision making require higher levels of reasoning. For this reason, Scholten (1996), states that the model is overly restrictive in assuming that cognitively complex changes in consumer/target audience attitudes are necessary for effective communication. Often, people do not use logic to think about issues at stake because perhaps they are distracted, unmotivated or too busy, so they use peripheral cues and heuristic shortcuts based on either visuals or persuasive knowledge and associations Myers (2002) and Scholten (1996). Besides, communication campaigns could still be effective without the target audience being guided through each and every step of the cognitive process.

Despite the above criticisms, the model is very useful and important for development purposes, especially when target audience are motivated and are capable of reasoning. In this state of mind, people take decisions through the central route, which are characterized by the steps described/prescribed in the model. The peripheral and central routes are the two cognitive paths for attitude change. But attitude along the central route are more persistent, more resistant to counter persuasion and more predictive of behaviour than attitude change along the peripheral route (Scholten, 1996). This being the case, it is therefore, of crucial benefit to make use of the model during media development to help guide the creation of rhetoric in graphic language employed to support development programmes.

Finally, the model underscores the necessity for communication actors, especially, the graphic encoder to make rhetoric graphic encoding choices that attract attention, stimulate interest, facilitate comprehension and encourage rapid adoption/utilization of development ideas. This would persuade and help target audience to change attitude as they make decision through the peripheral route and more especially through the central route of the cognitive path.

Having considered the input and the output variables the media team should have in mind in the treatment of graphic messages during media production process in order to produce rhetorical information, education, and communication materials, examples of graphic media that were produced and utilized for communication campaigns would be examined. This is in order to present the consequences of employing or neglecting the input and output variables during media development.

\section{Examples of Practical Rhetoric in Graphic Communication Campaigns}

The Delayed Sexual Debut Campaign, and the Use Condom Campaign in Nigeria, and the ORT Campaign in Egypt, are three nationwide communication campaigns on health issues that produced and utilized information, education and communication materials to persuade target audience to support development initiatives. These examples, sufficiently and necessarily depict attempts to generate rhetoric in graphic communication for development purposes, which employed or neglected the input variables of communication, and the output variables of persuasion.

The graphic media produced and utilized for the Delayed Sexual Debut Campaign is an epitome of virile rhetoric in graphic communication (see figure $2 \mathrm{a}$ and $\mathrm{b}$ ). During its media production process, the independent variables of communication and the dependent variables of persuasion were adequately employed.

The communication process started with the Source - Society for Family Health (SFH), which collaborated with the National Agency for the Control of AIDS (NACA). SFH and NACA, which are properly represented in the poster, are established non-governmental, and governmental organization respectively, however, SFH was active, while NACA was passive in this collaboration. These media requesting agencies are credible organizations with great concern on HIV/AIDS and other related issues on health. The power, control, concern, credibility, scurrility, expertise, trustworthiness are aptly stated in the Creative Brief (a document containing all activities concerning the campaign development process), Therein, it was concluded that there was a genuine need for the campaign in

Figure-2. A billboard poster on Sex is worth waiting for 'ZIP UP'; (a) English/Pidgin English Version; (b) Hausa Version; by Society for Family Health (Culled from Ebigbagha (2016)

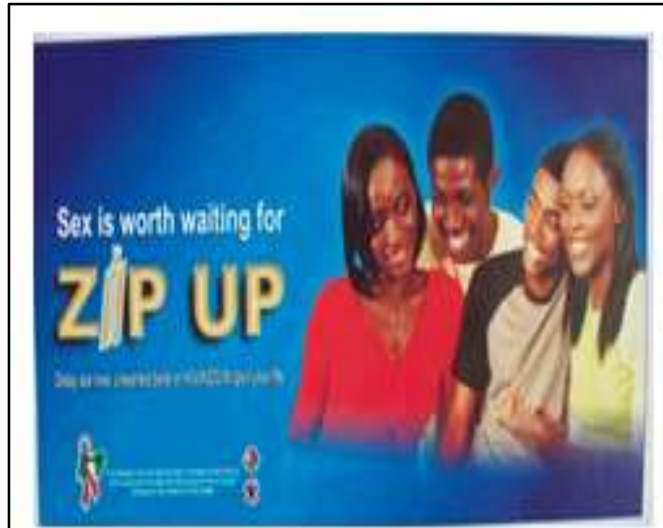

(a) EnglishPidgin English Version

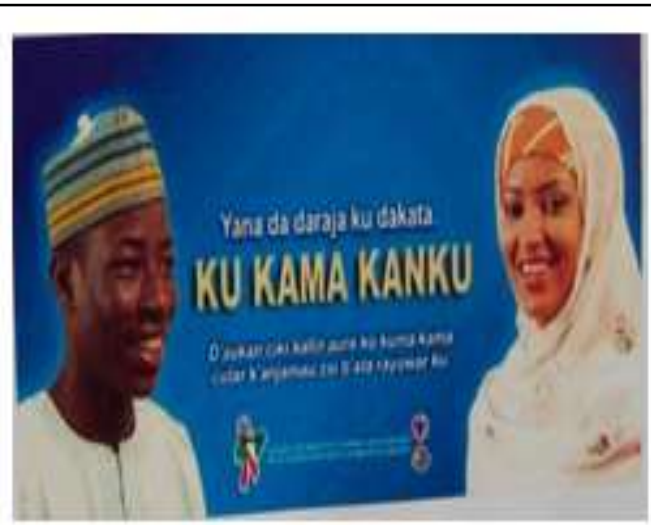

(b) Hausa Version 
Order to address prevalent unawareness and misconceptions about sexual debut, abstinence, and delay; and to empower those who wish to delay sexual activity until they are ready. This was suitably depicted in the billboard posters Also, the campaign was hinged on behavioral theoretical underpinnings, and employed all mass media distribution outlets in order to educate and persuade target audience. The television, and radio broadcasts were supported with billboards, posters and stickers characterized with graphic language that is attractive, familiar, and retaining its appeal of freshness to target audience. This was hinged on the interrogation of the input variables of the Source in communication with the output variables of persuasion that underscores the configuration of development messages in graphic forms and formats. The result is visual rhetoric based on heightening of awareness, exposure, attention, liking, comprehension, cognitive elaboration, skill acquisition, agreement, memory storage and retrieval, decision making, acting on decision, cognitive consolidation, and proselytizing.

The content of the message in the billboard posters of the Delayed Sexual Debut campaign are excellently organised. Its arguments that "sex is worth waiting for" because unwanted pregnancy can be ruinous. In addition, "ZIP UP" is an inclusion that is well chunked, emphasized and ordered to the left, this makes for attention, liking and comprehension. The concept of abstinence is a discrepancy to that of delay that was added with dexterity and without technical, semantic, syntactic or aesthetic uncertainty. Furthermore, the style with which the pictorial elements were combined with the verbal and other graphic elements are superb. It facilitated exposure, arrest attention, liking and other persuasive variables already mentioned. The ordering of the prime messages and the supporting information to the left and the pictorial elements to the right in the English/Pidgin English version (Figure 2a); and the verbal elements at the middle, framed with the pictorial elements to the right and left, in the Hausa version (Figure $2 b$ ) are diligently orchestrated following target audience conditions in the graphic encoding process of the stimulus conditions. Moreover, the logos of the Source (SFH, and NACA) are inclusions that were well positioned. The overall appearance was pleasant and free of any aesthetic or arousal uncertainty. Thus, a visual rhetoric was concocted that produced immense benefit, which minimized the incidence of sexually transmitted diseases, and unwanted pregnancy; and translated to a higher standard of living.

The input variables: arguments, inclusions, orderings, discrepancy, and style of the message in the billboard posters of the Delayed Sexual Debut campaign were thoroughly probed using the output variables of persuasion. For examples, how could the message - its content, be configured to get adequately exposed, attract attention, liked, understood, memorized, and cognitively elaborated by receivers? And how could the content of the message be transformed for target audience to acquire desired skill, support agreement, store and retrieve it from memory, make and act on decision, consolidate it cognitively, and proselytize as desired? Towards this end, the prime messages and supporting information are appropriately chunked: "ZIP UP", "Sex is worth waiting for", and "Unwanted belle fit spoil your life", were all suitably grouped and aligned/ordered to the left. Also, the pictorial elements were attractive, and appropriately represented the target audiences knowledge, aptitude and practices as well as reflecting their sociocultural environment. These interrogations on the message during media development process, created visual rhetoric, which was so successful that "ZIP UP", a prime message, became a catch phrase among target audience. The visual rhetoric produced desired communication outcome as purported.

In the Delayed Sexual Debut campaign, attention was paid to creating a virile set of channels that carry the message in different configurations, forms, and formats. Particular focus was devoted to modality, context, and nonverbal activities, functions and processes in making the channels utilized. This requires examination of the features and capabilities of the channel, its appropriateness and appeal to target audience as well as its potentials to suitably present the message as planned. Messages could be effectively communicated by both verbal and non-verbal channels; and channels that include variables, which are audio/visual, written/spoken, verbal/nonverbal, vocalic/visual non-verbal messages have different effect in various context. For this reason, both the big mass media such as radio, and television were employed in addition to small media such as billboard posters, pamphlets and flyers, among others. Examples includes Radio spots "Aunty Sabi says "Mosquito Do Not carry AIDS" and "Living positively with HIV/AIDS", which were radio programmes, and pamphlets respectively. The use of multiple channels are based on the need to subject development messages to afford maximum exposure, attention, liking, comprehension, cognitive elaboration, skill acquisition, agreement, memory storage, retrieval, decision making, acting on decision, cognitive consolidation, and proselytizing. This resulted in an effective delivery and distribution of messages that potently persuaded target audience with salutary effect, which was attested to by NARHS evaluation Ebigbagha (2016) citing Society for Family Health (2006).

The target audience conditions were critically taken into consideration in the graphic encoding process during media development of the IEC materials utilized in the Delayed Sexual Debut campaign. The demographics, personality, lifestyle and ability of the target audience are considered diligently following data from the NAHRS audience research. These input variables of the target audience, were examined in the light of exposure, attention, liking, comprehension, cognitive elaboration, skill acquisition, agreement, memory storage, retrieval, decision making, acting on decision, cognitive consolidation, and proselytizing. This is lucidly reflected in the English/Pidgin English version (Figure 2a), which afforded the knowledge, aptitude, and practices of target audience in the Southern part of Nigeria, where it is distributed; and in the Hausa version (Figure 2b) that support the knowledge, aptitude and practices in the Northern part of Nigeria, where it is distributed. The socio-cultural environment of the target audience were thoroughly examined and depicted, using appropriate language and suitable attires. The accuracy, brevity, and clarity that characterized the way the digital/verbal and iconic/pictorial signs in the posters are coalesced, give credence to the Source for properly regarding the target audience by paying attention to the input variables of scurrility, neoteny, expertise, credibility, and control, to mention a few. The result was a visual rhetoric 
with long salutary effect that afforded remembering and persuasion with desired change in target audience behaviour.

The Delayed Sexual Debut campaign was a huge success; the specific and general targets as well as the desired response from target response was achieved. The 'NARHS' and the 'NIGERBUS' evaluated the effectiveness of the campaign on HIV/AIDS, and found that the communication campaign reached its destination and was very effective (Ebigbagha, 2016).

Another example of activities, functions, and processes that culminated in the generation of practical rhetoric in graphic communication, is the development of the logo used for the ORT campaign in Egypt (see Figure 3a, b, and c).

Figure-3. a, b and c: Logos Pre-tested for the ORT Campaign in Egypt, showing how test improved logo (Culled from World Health Organisation (1987)

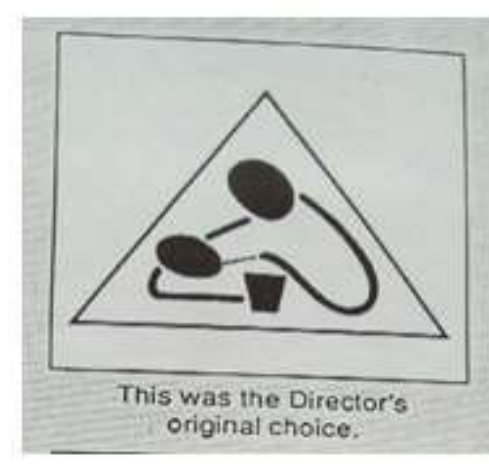

(a)

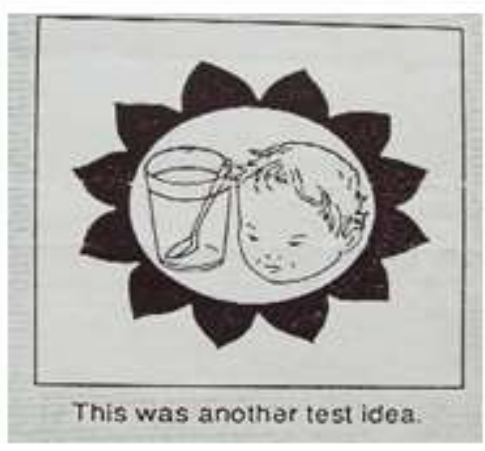

(b)

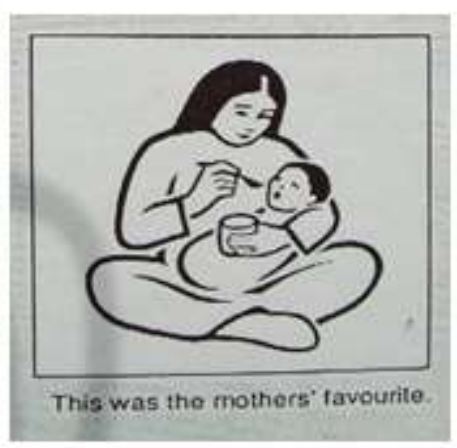

(c)

The ORT Campaign in Egypt, emphasises strongly the need to thoroughly regard the input variables of communication along with the output variables of persuasion in order to produce effective rhetoric in graphic communication. It underscores the activity of proper evaluation of target audience conditions and socio-cultural environment through pre-test, which is paramount to avoid rash decision-making and production of ineffective graphic communication.

The first Executive Director of the Egypt ORT Project was to decide on and adopt a logo for the project. Having examined many logo ideas that were submitted to the project by different artists and designers, he liked one more than the others (Figure 3a). The director was tempted to adopt the particular logo he liked for the project without further deliberation. However, he instinctively decided to withhold his opinion or most preferred choice until all logos were thoroughly tested with a sample of the target audience - mothers.

The result reveals that the logo, which the director most preferred, was the least favoured by the pre-test respondents. Also, it shows that the logo chosen needed to be revised in order to be free from the semantic and syntactic uncertainties that bedeviled it (Figure 3c). A large number of the respondents suggested that the colour need be modified, and the mother pictured in the logo should wear a wedding ring.

The result was a practical rhetoric in graphic language, which was adopted for the ORT campaign. The experience was so impressive to the Director that he often recounted the story, to reiterate the need to evaluate draft with target audience for successful communication in development programme. Had the director adopted the particular logo he liked without the target audience involvement in the development process through pre-test, the necessary improvements made on the logo, would not have been possible. The addition of a wedding ring on the finger of the pictured mother in the logo, is very significant and instructive. It pinpoints the socio-cultural role in the determination of target audience perception, interpretation, and response to graphic communication.

The 'Use Condom' communication campaign, is an example of efforts towards production of practical rhetoric that failed because it neglected the described/prescribed utilization of independent variables of communication and dependent variables of persuasion. The IEC material examined are a poster and a sticker (Figure 4 a, and b) respectively. 


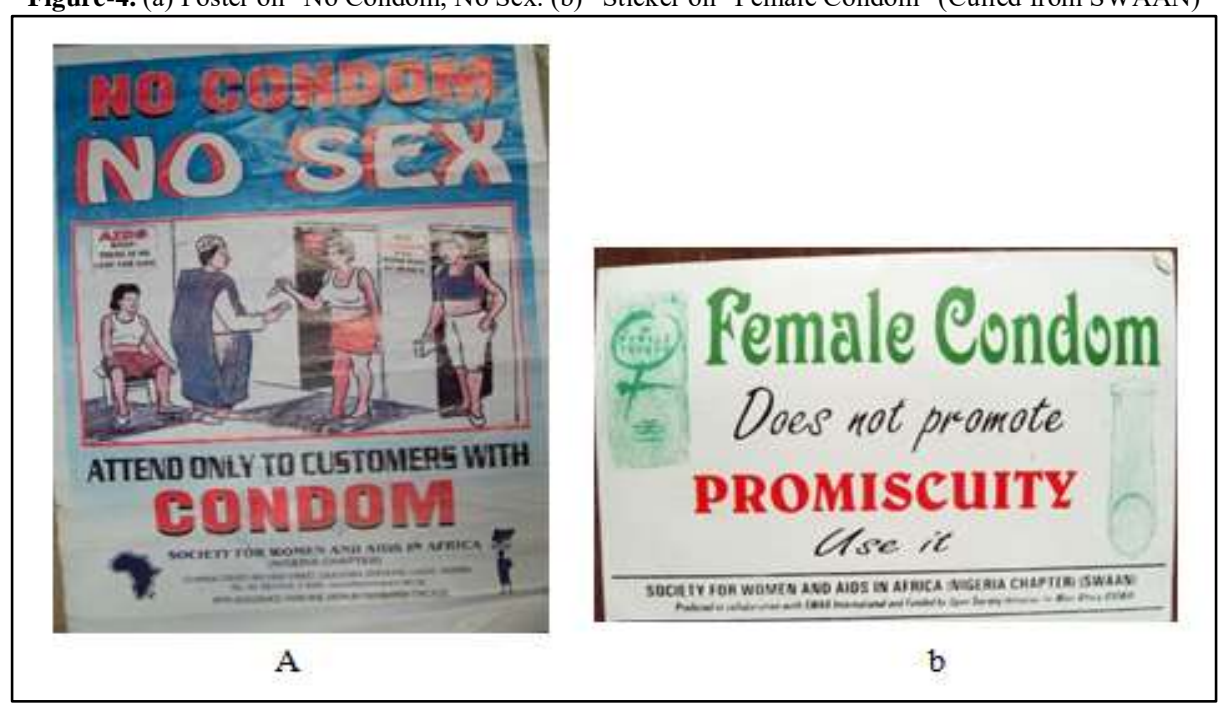

The main aim of this poster (Figure 4a), was to emphasis the use of condom to warn against unprotected sex in order to stem the HIV/AIDS epidemic. It depicts a man approaching three ladies in a brothel in an attire commonly worn by Western and Northern Nigerians. Although the poster was fairly illustrated, it afforded connotative meanings that could ruin achievement of the desired overall communication goal. These inferences include: only those who go to brothel, patronise prostitutes, from the Western and Northern parts of Nigeria, or want to prevent HIV/AIDS are at risk of the virus and need use condom. These faulty interpretations would have been structured to what it should purportedly denote, had the input variables of communication been adequately examined with the output variables of persuasion. Scurrility, expertise, control, inclusions, omissions, and modalities, among other independent variables, when interfaced with exposure, attention and liking, to mention a few of dependent variables, would have resulted in effective visual rhetoric. The attire of the man would have been revised for socio-cultural reason. Also the attention to brothels and prostitutes would have been revised because the most liable group to HIV/AIDS (fifteen to twenty-five years) are more in the schools and out-of-school environment than in the brothel; and prostitution along the street is now common and fashionable than the brothels.

Also, the sticker, 'Female Condom Does Not Promote Promiscuity Use It' (Figure 4b) that was produced and used for the use of condom was ineffective. It emphasized 'Promiscuity', which is in red hue and boldly expressed in capital letters. The prime message 'Female Condom' is de-emphasized in green hue and boldly written with initial capital and low letters. The supporting information 'Does not promote' and 'use it' are written in slim italics with black. The pictorial image of the condom and the container are oppositely placed on both sides in half tone green. Each set of words are expressed in a different typeface and in all, there are four typefaces. On the whole, the haphazard placement of both the graphic and typographic elements defies any existing described/prescribed layout for the production of graphic language. At a glance from a short distance, these three words 'Female Condom Promiscuity' are conspicuous; so, the sticker seems to encourage promiscuity, which is against its real communication goal. Besides, it was unattractive, uninteresting and filled with semantic, syntactic, and aesthetic/arousal uncertainties. It failed to elicit the desired response from target audience because they could neither understand nor identify it.

\section{Findings}

Adequate consideration and application of input variables of communication in conjunction with output persuasion variables by the graphic encoder among other members of the media team during media development process is needful to produce practical rhetoric in graphic communication.

Rhetoric in graphic language is a product of adequate collaboration between communication actors during the media production process. This requires coalesce of the Source's credibility, trust, expertise, scurrility, concern, control, power, similarity, familiarity, and neoteny with target audience demographics, personality, ability, knowledge, aptitude and practices while structuring and encoding development messages into graphic media or channels. In addition, the encoding process need take into consideration the content of the message, its arguments, inclusions, orderings, discrepancy, and style as well as the modality, context, and nonverbal features of the channel. When these input variables of communication are orchestrated in the light of the mediation variables of persuasion exposure, attention, liking, comprehension, cognitive elaboration, skill acquisition, agreement, memory storage, retrieval, decision-making, acting on decision, cognitive consolidation, and proselytizing; the result is often satisfactory.

When the communication actor examines the stimulus conditions in the light of target audience conditions, and tailors both to suit the potentialities of channels, target audience usually responds as desired. This underscores the generation of persuasive messages in graphic communication campaigns for development. 


\section{Conclusion}

Practical rhetoric in visual language is pivotal for the success of human development, and a virile vehicle for education, mobilization, and persuasion of target audience to participate in development programmes.

The generation of rhetoric in graphic language is hinged on thorough consideration of the source, message, channel, target audience, and destination input variables of communication vis-à-vis the output variables of persuasion, and bereft of technical, syntactic, semantic, pragmatic, and aesthetic/arousal uncertainties. This usually results in efficacious graphic communication, while neglect, result in communication failure.

Therefore, graphic encoders/designers, other media team members, media requesting agencies, and stakeholders in development, need to be conversant with, internalize, and utilize coalesce of input communication, and output persuasion variables. This is indispensable to producing visual rhetoric that enlist target audience support for development initiatives with salutary effect.

\section{References}

Anonymous (2007). Introductory models and basic concepts. The Lasswell Formula: Lasswell. http://wwwgoogle

Blair, M. G., Jones, S. R. and Simpson, H. R. (1975). Educational psychology. Macmillan Publishing Co; Inc: New York, U.S.A.

Bowers, J. (1973). Action research and the production of communication media, in r. Varma, s.L. Ghosal, j. Bowers, and r.H. Hull (eds), action research and the production of communication media. Report on the all field workshop on action research in agricultural information communication, udaipur. Typographic Unit, University of Reading: England.

David, L. (2015). Paradigms in learning theories. Available: https://www.learningtheories.com/paradigms

Ebigbagha (2016). Graphic encoding choices in development communication: Identifying the several loops of the graphic encoder/media team collaboration. Lap Lambert Academic Publishing - Ominiscriptum: Gmb and Co. KG Germany.

Ebigbagha, 2016b. "Graphic communication for sustainable development: Eliciting desired response from target audience." In Ebohon, O.J., Ayeni, D.A., Egbu, C.O., and Omole, F.K, Process of the Joint International Conference (JIC) on 21st Century Human Habitat: Issues, Sustainability and Development, 21-24 March 2016, Akure, Nigeria. pp. 1045-52.

Ebigbagha (2019). The laswell formula: Implications for graphic communication in development initiatives. Journal of Ceramics and Environmental Design, 6(2): 215-32.

Ejembi, E. A. (1989). Graphic communication in development programmes: Models for communicators. An Unpublished Ph.D. Thesis, Department of Typography and Graphic Communication, University of Reading, England.

Lidwell, W., Holden, K. and Butler, J. (2013). Universal principles of design. Rockport. Massachusetts, USA.: Beverly.

Machado, M. (2002). Information processing and other hierarchy of effects theories. 382j: Theories of persuasive communication and consumer decision making. University of Texas at Austin, Fall 2002. corbis.com.: http://www.google.ie

McGuire, W. J., 1989. "Theoretical foundations of campaigns." In Rice, R.E.; and Atkin, C.K. (ed.), Public Communication Campaigns; Sage Publications, Newbury Park, California, U.S.A.

McGuire, W. J. (1999). Constructing social psychology: Creative and critical processes. Cambridge University Press: Cambridge, England.

Myers, D. G. (2002). Social psychology. McGraw-Hill: New York, U.S.A.

Scholten, M. (1996). Lost and found: The information-processing model of advertising effectiveness. Journal of Business Research, 37(2): 97-104.

Society for Family Health (2006). Corporate presentation. Society for Family Health: Abuja, Nigeria.

Watt, J. H., 1979. "Television form, content attributes, and viewer behaviour." In Voigt, J.M., and Hanneman, J.G. (ed.), Progress in Communication Sciences, Volume I, Ablex Publishing Corporation, Norwood, New Jersey, U.S.A.

World Health Organisation (1987). Communication - a guide for managers of national diarrhoeal disease control programmes. World Health Organisation: Geneva, Switzerland.

Wright, P. (1981). Problems to be solved when creating usable documents. MRC Applied Psychology Unit: Cambridge, England. 